\title{
Comparison of Partial Least Squares and Artificial Neural Network for the prediction of antioxidant activity in extract of pegaga (centella) varieties from 1H Nuclear \\ Magnetic Resonance spectroscopy.
}

\begin{abstract}
Multivariate data analysis of $1 \mathrm{H}$ Nuclear Magnetic Resonance spectra was applied for the prediction of antioxidant activity in five different Pegaga (C. asiatica (var 1), C. asiatica (var 2), C. asiatica (var 3) H. bonariensis and H. sibthorpioides) varieties. Linear (Partial Least Square regression) and non linear (Artificial Neural Network) models have been developed and their performances were compared. The performances of the models were tested according to external validation of prediction set. The result showed that the Partial Least Square model provided better generalization than Artificial Neural Network. Despite those, both models are considered reasonably acceptable. Regression coefficient and VIP values of the PLS model revealed that 3,5-O-dicaffeoyl-4-O-malonilquinic acid (irbic acid), 3,5-di-Ocaffeoylquinic acid, 4,5-di-O-caffeoylquinic acid, 5-O-caffeoylquinic acid (chlorogenic acid), quercetin and kaempferol derivatives are the components responsible for the antioxidant activity. In addition, the spectroscopic pattern of the Pegaga varieties, as shown by the PLS score plots was consistent with the corresponding antioxidant activity. Prediction of the antioxidant activity from $1 \mathrm{H}$ NMR spectra using this approach is useful in assessing the quality of medicinal herb extracts.
\end{abstract}

Keyword: Centella asiatica; Anti-oxidant activity; 1H Nuclear Magnetic Resonance; Partial Least Square regression; Artificial Neural Network. 